\title{
Light Propagation in a Curved-Space Friedmann Universe
}

\author{
S. J. Prokhovnik
}

School of Mathematics, University of N.S.W., Kensington, Australia, 2033

\author{
A. E. Paparodopoulos \\ 3 Perikleous Avenue, 155-61 Athens, Greece
}

Z. Naturforsch. 49a, 543-546 (1994); received February 7, 1994

\begin{abstract}
It was shown [1] that, in a Friedmann universe considered as an expanding ensemble of fundamental particles and whose 3-space is Euclidean, the propagation of light and its associated wavelength(s) partake in the expansion of the universe. In consequence, a light-signal emitted from any source will reach and pass every fundamental particle in its path at the same speed, $c$, despite the systematic mutual recession of any pair of such particles. This result, derived from the Robertson-Walker metric, is in full accord with all local physical experience of light transmission and with the requirements of Einstein's Special Theory of Relativity. It is shown here that the same result also applies to any curved-space Friedmann universe.
\end{abstract}

Key words: Cosmology, Light propagation, Special Relativity.

\section{Basic Assumptions}

In this treatment of light propagation in a curvedspace Friedmann universe we shall lean on the same basic assumptions and their associated metric as employed in [1] and, indeed, as in most texts dealing with cosmological theory. This involves treating the universe as an ensemble of "fundamental particles" (corresponding 'ideally' to the galaxies and their clusters of our observable universe), in mutual recession according to Hubble's Law, $w=H r$, and governed by a Cosmological Principle. We may imagine a "fundamental observer" associated with each fundamental particle.

The Robertson-Walker metric gives mathematical expression to the cosmological model described as above; it employs co-ordinates, say $(\underset{\sim}{r} \underset{\sim}{\theta}, \phi)$, which describe the locations of the fundamental particles/observers in relation to one of these treated as at the origin of the (fundamental) frame defined by the metric. An essential feature of the metric is that the space co-ordinates (of any fundamental particle) are time-invariant, that is they are considered as comoving with the expansion of the ensemble and its associated expanding fundamental frame. Note that, in accordance with the Cosmological Principle, any fundamental

Reprint requests to Professor S. J. Prokhovnik, School of Mathematics, University of New South Wales, PO Box 1, Kensington, NSW 2033, Australia. particle can be considered as at the centre of our model universe, and hence treated as at the origin of this frame; the metric is invariant with respect to choice of such origin.

The metric incorporates a curvature parameter, $k$, whose possible values, $0,+1$, or -1 correspond to the assumed 3-space curvature of the model, and a "scalefactor", $R(t)$, which describes the nature of the expansion in terms of the "cosmic time" measure, $t$ defined in [1]. Note that the fundamental frame, defined by the metric, is a unique cosmologically-based reference frame, associated with the large-scale distribution of matter and energy (e.g. radiation) in the observable universe. It is now also observationally definable in two ways as described in [1].

The null-geodesic of the Robertson-Walker metric, corresponding to a radial light-path, may be expressed, as shown in [1] by

$$
\pm c \mathrm{~d} t=\frac{R(t) \mathrm{d} r}{\left(1+\frac{1}{4} k r_{\sim}^{2}\right)}
$$

the choice of sign depending on whether the origin of the reference frame is taken as at one end of the lightpath or the other. If we take the origin of the frame at the locality of the source of a light-signal, then the plus sign applies; and the minus sign is applicable if the locality of an observer of the light is taken as the origin of the frame. Both approaches are useful, depending on our purpose, but they must of course yield results which are mutually consistent since the metric and its 
consequences must be observationally equivalent with respect to all fundamental observers.

\section{Implications of the R.-W. Null-geodesic for a Curved-Space Universe}

The argument for this case, presented in [1], led to a result which was internally inconsistent (it differed with choice of origin) and violated the Cosmological Principle. A close consideration of the procedure employed in [1] revealed an oversimplification of the basic data, and pointed to a revised procedure which dealt with the data more faithfully. The revised deduction has its own intrinsic interest and highlights the employment of dimensional logic to relate the comoving radial co-ordinate, $\underset{\sim}{r}$, with distances and speeds, etc., of direct concern to observational cosmology and astronomical data.

Thus consider, as before in [1], a light-signal emitted at (cosmic) time, $t_{0}$, from a (fundamental particle) source, $F_{0}$, treated as at the origin of the R.-W. reference frame. In the context of a hyperbolic 3-space universe for which $k=-1$, the path of the light-signal is described by the null-geodesic (1), in the form

$$
c \mathrm{~d} t=\frac{R(t) \mathrm{d} r}{1-{\underset{\sim}{r}}^{2} / 4} .
$$

Now, the Robertson-Walker metric derives from the metric of General Relativity applied to the conditions of a model of the universe and its underlying assumptions. In turn, Einstein's field equations also place constraints on this model in a manner expressed by the Friedmann equation. As shown in [1], this equation restricts the possible nature of the scale factor, $R(t)$, for each of the three values of $k$. For $k=-1, R(t)$ is required to satisfy the pair of parametric equations

$$
\left.\begin{array}{l}
R=\alpha(\cosh q-1) \\
c t=\alpha(\sinh q-q)
\end{array}\right\},
$$

where $\alpha$ is a constant associated with features of the cosmos and has the dimension of length (or distance) and $q$ is a parameter such that $R=0$ and $t=0$ when $q=0$. Note that $R$ and (of course) $c t$ also have the dimension of a length. (In the corresponding argument, presented in [1], an oversimplified view of $R(t)$ was employed.)

Reverting to the result (2), we need now to employ a dimensionality argument which requires that only terms of the same dimension(s) can be equated or added, etc. Hence in (2), ${\underset{\sim}{r}}^{2}$ and so also $\underset{\sim}{r}$ and $\mathrm{d} \underset{\sim}{r}$ must be dimensionless, that is equivalent to a pure number; and since $c \mathrm{~d} t$ is equivalent to an element of length, $R(t)$ must then also have the dimension of a length a condition which is satisfied by the formulation of $R(t)$ in (3). We can now define a distance, $r$, from $F_{0}$, associated with the value of $\underset{\sim}{r}$ for any fundamental particle by considering a radial light-signal which passes two neighbouring fundamental particles at epochs $t$ and $t+\mathrm{d} t$. Then the element of distance, $c \mathrm{~d} t$, travelled by the light-signal between these epochs must be equal to the space-interval element, $\mathrm{d} r$, separating these particles at the time, $t+\mathrm{d} t$. It then follows from the result (2) that

$$
\mathrm{d} r=\frac{R(t) \mathrm{d} r}{1-\stackrel{\sim}{r}^{2} / 4},
$$

and on integrating we obtain

$$
r=R(t) \ln \left(\frac{1+\underset{\sim}{r} / 2}{1-\underset{\sim}{r} / 2}\right),
$$

at any epoch of cosmic time, $t$, and since $r=\underset{\sim}{r}=0$ when $t=t_{0}$. [Note that if $k=0$, it is sufficiently valid to define $r$ by " $r=R(t) \underset{\sim}{r}$ ", which implies that $\mathrm{d} r=R(t) \mathrm{d} r$ at any time, $t$. However, the second of these expressions is more fundamental since it relates directly to the formulation of the null-geodesic, and so is the only one applicable for all values of $k$.]

If follows from (5) that the speed of recession, $w$, relative to $F_{0}$, of any fundamental particle, $F$, is given by

$$
w=\dot{r}=\dot{R}(t) \ln \left(\frac{1+\underset{\sim}{r} / 2}{1-\underset{\sim}{r} / 2}\right)
$$

since the comoving co-ordinate, $\underset{\sim}{r}$, is, of course, timeinvariant. Also, the distance, $s$, travelled by a light-signal relative to its source, $F_{0}$, when it reaches a fundamental particle, $F$, at $(r, \underset{\sim}{r})$ is given by (5), since

$$
s \equiv r=R(t) \ln \left(\frac{1+\underset{\sim}{r} / 2}{1-\underset{\sim}{r} / 2}\right) .
$$

But

$$
\dot{s}=R(t)\left[\frac{\dot{r}}{1-\sim_{\sim}^{r} / 4}\right]+\dot{R}(t) \ln \left(\frac{1+\underset{\sim}{r} / 2}{1-\underset{\sim}{r} / 2}\right),
$$

since here $\underset{r}{r}$ varies as well as $t$, as the light passes successive fundamental particles; and noting that $\frac{\mathrm{d}}{\mathrm{d} t}=\frac{\mathrm{d} r}{\mathrm{~d} t} \frac{\mathrm{d}}{\mathrm{d} r}=\underset{\sim}{\dot{r}} \frac{\mathrm{d}}{\mathrm{d} r}$. And invoking the results (2) and (6) in (7), we obtain

$$
\dot{s}(r, t)=c+w(r, t),
$$


exactly as obtained in [1] for a Euclidean 3-space universe for which $k=0$. An equivalent result, $\dot{s}=-c+w$, obtains (as for the $k=0$ model) if a fundamental particle at the locality of an observer in the path of the light-signal is taken as at the origin of the R.-W. frame.

For a cosmological model whose 3-space is ellipsoidal so that $k=+1$, the same procedure as employed above may be used to deduce the implications of the null-geodesic appropriate for this model; and the same results as (8) and etc. are found to apply for this case also. These results imply that the speed of light, from any source, is precisely $c$ relative to a fundamental particle/observer at any locality of the universe, as required by the Cosmological Principle; they are also in accord with all physical observation, as detailed in [1].

To obtain further results we need to express $s(=r)$ and $w$, etc. in terms of the parameter $q$. Combining (5) and (6), we obtain

$$
w=\dot{r}=\frac{\dot{R}(t)}{R(t)} r=\frac{\dot{R}(t)}{R(t)} s,
$$

a form of Hubble's Law. Hence (8) may be expressed by

$$
\dot{s}=c+\frac{\dot{R}(t)}{R(t)} s,
$$

which has the solution

$$
s=R(t) \int \frac{c \mathrm{~d} t}{R(t)} .
$$

Then invoking the formulation of $R(t)$ in (3), and its consequences

$$
\begin{aligned}
& c \frac{\mathrm{d} t}{\mathrm{~d} q}=\alpha(\cosh q-1), \\
& \text { so that } \dot{R}(t)=\frac{\mathrm{d} R / \mathrm{d} q}{\mathrm{~d} t / \mathrm{d} q}=c \operatorname{coth}(q / 2),
\end{aligned}
$$

(10) yields

$$
s=R(t) \int \mathrm{d} q=\alpha(\cosh q-1)\left(q-q_{0}\right),
$$

since we may take $q=q_{0}$ when $s=0$ at $t=t_{0}$. Hence, combining (9) and (12), and invoking (3) and (11), we obtain

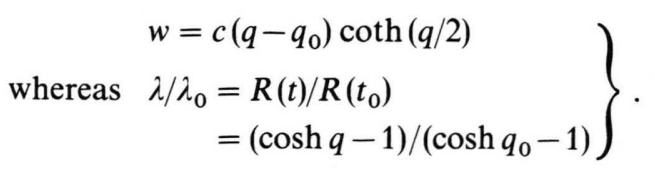

Equation (13) represents the relationship between the observed redshift, $z\left(=\lambda / \lambda_{0}-1\right)$, and the recession speed, $w$, of its cosmic source; it enables us to find the value of $w$ corresponding to the value of $(1+z)$ for any given $q$ and $q_{0}$, and hence for any given redshift. Thus, taking $q_{0}=0.10$ and $q=0.11$, and using (13) we obtain

$$
w=0.18 c
$$

and

$$
1+z=\lambda / \lambda_{0}=1.21, \text { whereby } z=0.21 .
$$

It is interesting to compare this result with the corresponding result for an Einstein-de Sitter universe. For this model, as shown in [1],

$$
\lambda / \lambda_{0}=(1-w / 2 c)^{-2} ;
$$

hence, for $w=0.18 c, \lambda / \lambda_{0}=1.21$, also! In general, for any Friedmann model, $\lambda / \lambda_{0}=1+\mathrm{z} \approx 1+w / c$, for small $w$.

\section{Final Results}

The result (8) confirms that light-propagation partakes in the expansion of a curved 3-space Friedmann universe in the same manner as it does for a 'flat' $(k=0)$ Friedmann universe. Hence, the discussion in [1], dealing with the significance of this result and its agreement with our understanding of light-propagation on the local scale, applies to all Friedmann-type cosmic models.

However, in one respect the curved 3-space Friedmann models do differ from the flat 3-space models. For the former models, the linear formulation, $w=H(t) r$, of "the Hubble Law" does not apply. The deviation from linearity can be seen as a consequence of the definition (4), whereby equal intervals, $\underset{\sim}{\boldsymbol{r}}$ (in terms of the comoving co-ordinate, $\underset{\sim}{r}$ ), between neighbouring galaxies of a succession of such do not correspond to equal intervals, $\Delta r$, in terms of the distance (from the origin) co-ordinate $r$. Hence, for non-Euclidean Friedmann models the $w-r$ relation would need to be expressed by $w=H(t, r) r$, resulting in a deviation from linearity which increases with distance.

Our approach involves the employment of terms, $r, s, w$, etc. and the deduction of their relationship with observational data, of direct relevance to the astronomical study of cosmic sources of light and to the estimation of the luminosity distance and the redshift- 
related recession speed of such sources. By contrast, conventional outlines of cosmological theory employ only the comoving co-ordinates and the cosmic time, $t$, and so present counterparts to our results which are difficult to apply by observational cosmologists.
Finally, one must again insist that the notion of a fundamental reference does not violate the validity of Special Relativity. Indeed, as shown in [1], the existence of such a frame enhances the understanding of Einstein's Theory.

[1] S. J. Prokhovnik, Z. Naturforsch. 48a, 915 (1993). 